\title{
Analysis of wave generators and absorbers in basins
}

\author{
by J. N. Newman
}

May 8, 2008

\begin{abstract}
Wave generation and absorption are analyzed in a closed basin, within the framework of linear potential theory. Wavemakers on the sides of the basin are used for both generating and absorbing the waves. Relations are derived governing the control of the absorbers, to reduce or eliminate reflected waves from the walls of the basin. These relations are tested by showing numerical examples of wave systems in square and circular basins, first without a body in the basin and then with a floating hemisphere. Computations of the added mass, damping, and exciting forces on the hemisphere are presented to indicate the effectiveness of the wave absorbers. Two-dimensional results are shown in the time domain to illustrate some of the transient effects associated with different types of absorber controls.
\end{abstract}

\section{Introduction}

Experimental wave basins are used to measure wave effects on various types of structures and vessels, including models of ships, offshore platforms, and other bodies. The usual objective is to simulate the open-water environment where the free surface is unbounded, except by the body (or by multiple bodies). In order to achieve this objective it is necessary to eliminate or minimize hydrodynamic effects associated with the finite dimensions of the basin. Wall effects which are not related directly to waves are negligible if the basin is sufficiently large relative to the body length scale. However the effects associated with wave reflection from the walls will persist regardless of the size of the basin. Thus it is essential to both generate and absorb waves in a controlled manner, to simulate the open-water conditions.

Square or rectangular wave basins are usually equipped with banks of wavemakers on one or two sides. Beaches are used on the opposite sides to avoid or minimize reflected waves. Most beaches consist of sloping porous surfaces which absorb the wave energy through a combination of viscous dissipation and breaking. Partial reflection from beaches is unavoidable. In some cases it is necessary to limit the time of an experiment so that the reflected waves do not affect the test area of the basin.

As an alternative to passive beaches, wavemakers with suitable controls can be used as absorbers $[1,2,3]$. We shall refer here to 'wavemakers' as devices which can be used either to generate or absorb waves, or to perform both functions simultaneously. Absorbing wavemakers have several advantages relative to conventional beaches. At least in linear theory, low or zero reflection can be achieved, and less space is required in the experimental domain of the basin. Another advantage is that waves which are reflected back toward the generators can be absorbed. Absorbing wavemakers can also be used to reduce the time required for residual 
wave attenuation between experimental runs. In addition to their use in physical wavetanks, absorbing wavemakers are also used in numerical wavetanks $[4,5]$.

The linear analysis of wavemakers and absorber control systems is relatively straightforward in two dimensions, but the situation is more complicated if the wave motion is threedimensional. In order to generate and absorb plane waves at oblique angles it is necessary to use a large number of wavemaker elements with small widths compared to the wavelength [6]. A more fundamental problem is to control the absorbers in an effective manner for oblique or multi-directional waves.

Wavemakers can be analyzed with Havelock's theory [7], which applies to a semi-infinite fluid domain. In the case of generators a radiation condition is imposed, and each wavemaker radiates waves which propagate to infinity. For absorbers the same theory applies, but with the waves moving in the opposite direction. As in the case of a floating body in an infinite fluid, the hydrodynamic pressure force acting on each element includes both added-mass and damping components. This approach is intuitively logical if the horizontal scale of the basin is large compared to the wavelength, and if reflections are ignored.

It is more rational to consider the basin as a finite domain, but this changes the linear inviscid theory in a fundamental manner. Waves are reflected on the basin walls, and there is no energy radiation. Considering each wavemaker mode separately, and assuming steady-state harmonic time dependence, standing waves are generated and the fluid velocity throughout the basin oscillates with the same phase. There is no wave damping, and the pressure force acting on each wavemaker element can be expressed completely in terms of an added-mass matrix. Nevertheless progressive waves can be generated, with 'effective' damping and energy absorption, by combining the motions of different wavemaker elements with appropriate phase differences.

In this paper linear theory is used to study the performance of wavemakers in basins. We restrict our attention to basins with a square or circular planform, and to hinged wavemakers with vertical surfaces which rotate about a submerged horizontal axis. Similar results can be expected for other planforms and wavemakers.

The first problem considered is that of generating and absorbing a plane progressive wave system without the presence of a body. The theoretical framework is outlined in Section 2. Wavemakers and conditions for optimizing absorbers are considered for two-dimensional basins in Section 3, for oblique waves in Section 4, and for three-dimensional basins in Section 5. The extension to include a body is considered in Section 6, where it is shown that close approximations to the open-water forces on a floating body can be achieved with suitable control of the absorbers. In Section 7 two-dimensional wavemakers are analyzed in the time domain, to compare the transient effects of different absorber strategies. Section 8 gives a brief description of the computational methods, and Section 9 includes discussion and conclusions.

\section{Theoretical Analysis}

Cartesian coordinates $x, y, z$ are used, with $z=0$ the plane of the equilibrium free surface and the $+z$-axis directed upwards. The fluid depth $h$ is constant, with the bottom at $z=-h$. The sides of the basin are assumed to be vertical, and covered by an array of $N$ wavemakers. The fluid domain is enclosed by the boundary surface $S$ including the wavemakers and bottom of the basin, and by the free surface. The normal vector $\mathbf{n}$ is directed out of the fluid domain on $S$. The complex time-factor $\mathrm{e}^{\mathrm{i} \omega t}$ is assumed throughout, except in Section 7.

If the $j$ 'th wavemaker oscillates with complex amplitude $\xi_{j}$, the velocity potential can be 
defined as

$$
\phi=\mathrm{i} \omega \sum_{j=1}^{N} \xi_{j} \phi_{j}
$$

Here $\phi_{j}$ is the radiation potential for each wavemaker, satisfying the boundary condition

$$
\frac{\partial \phi_{j}}{\partial n}=n_{j}
$$

on the submerged surface $S_{j}$ of the $j$-th wavemaker, where $n_{j}$ is the normal displacement when $\xi_{j}=1$. If the wavemakers are hinged about the bottom,

$$
n_{j}=\frac{z+h}{h} \text { on } S_{j} .
$$

The normal velocity $\partial \phi_{j} / \partial n=0$ on the other wavemakers, and on the bottom of the basin. It is convenient to define $n_{j}=0$ everywhere on $S$ except on $S_{j}$, so that (2) applies everywhere on $S$. The normal displacement $n_{j}$ is real, with the same phase at all points on the wavemaker. (More generally, in cases where the motion of a wavemaker is complex, it can be decomposed into two real modes.)

The linear free-surface condition on $z=0$ is

$$
\omega^{2} \phi_{j}-g \frac{\partial \phi_{j}}{\partial z}=0
$$

where $g$ is the gravitational acceleration.

The generalized pressure force acting on the $i$ 'th wavemaker is

$$
F_{i}=\iint_{S} p n_{i} d S=-\mathrm{i} \rho \omega \iint_{S} \phi n_{i} d S=\rho \omega^{2} \sum_{j=1}^{N} \xi_{j} \iint_{S} \phi_{j} n_{i} d S
$$

where the linear form of Bernoulli's equation is used to evaluate the pressure $p, \rho$ is the fluid density, and there is no contribution from the hydrostatic pressure since the wavemaker's normal displacement is horizontal. In the present case $F_{i}$ is equivalent to the moment about the hinge axis.

Since the fluid domain is bounded and the only inhomogeneous boundary condition is real, it follows that $\phi_{j}$ is real and the generalized pressure force acting on the $i$ 'th element can be expressed in the form

$$
F_{i}=\omega^{2} \sum_{j=1}^{N} \xi_{j} A_{i j}
$$

where

$$
A_{i j}=\rho \iint_{S} n_{i} \phi_{j} d S
$$

is the added-mass matrix.

We shall assume that the principal objective is to generate a progressive 'incident' wave system of amplitude $A$, represented by the potential

$$
\phi_{I}=\frac{\mathrm{i} g A}{\omega} \frac{\cosh k_{0}(z+h)}{\cosh k_{0} h} \mathrm{e}^{-\mathrm{i} k_{0}(x \cos \beta+y \sin \beta)}
$$

where $\beta$ is the angle of propagation relative to the $+x$-axis. The wavenumber $k_{0}$ is the positive real root of the dispersion relation

$$
\frac{\omega^{2}}{g}=k \tanh k h
$$


The wave system (8) can be produced in a basin with no obstructing bodies if the normal velocity everywhere on the walls of the basin is equal to $\partial \phi_{I} / \partial n$. This ideal condition can be approximated if the wavemaker width is sufficiently small relative to the wavelength $2 \pi / k_{0}$, and the amplitude of each element is defined by

$$
\xi_{j}=\left(n_{x} \cos \beta+n_{y} \sin \beta\right) \mathrm{e}^{-\mathrm{i} k_{0}(x \cos \beta+y \sin \beta)} .
$$

Here $\left(x_{j}, y_{j}\right)$ are the horizontal coordinates of the wavemaker centroid and $\left(n_{x}, n_{y}\right)$ are the components of the normal vector.

The condition when (10) is used to define the wavemaker amplitudes a priori will be referred to as 'kinematic' absorption. The principal approximation in this scheme is to neglect evanescent modes which are only significant close to the wavemakers, a region that usually is not used for experiments.

Kinematic absorption is not appropriate if there are disturbances of the incident wave system, due to radiation and scattering from bodies within the basin or any other cause. In experimental applications it is customary to control the absorbers based on measured values of either the incoming waves, as in [1], or the hydrodynamic force acting on the wavemaker, as discussed in [2] and [3]. Following the latter approach, we shall consider 'dynamic' absorption where each absorber responds to the pressure force it experiences in accordance with the equation of motion

$$
\sum_{j=1}^{N} A_{i j} \xi_{j}+\left(m_{i}-\mathrm{i} d_{i} / \omega\right) \xi_{i}=0 .
$$

Here $m_{i}$ and $d_{i}$ represent the inertial and damping coefficients of a linear control system which restrains the absorber $i$. If a subset of the wavemakers $\left(1 \leq j \leq N_{A}\right)$ act as absorbers and the others $\left(N_{A}+1 \leq j \leq N\right)$ as generators, (11) is applied with $\left(1 \leq i \leq N_{A}\right)$, to solve for the absorber amplitudes, and the amplitudes of the generators are defined by (10).

\section{Two-dimensional wavemakers}

It is useful to consider the case of two-dimensional motion with two identical wavemakers at the opposite ends of a rectangular basin. The first wavemaker, say at $x=0$, oscillates with amplitude $\xi_{1}$ and radiates waves in $x>0$. Neglecting the evanescent near-field motion the potential on the free surface is represented in the form

$$
\xi_{1} \phi_{1}(x, 0) \simeq \xi_{1} \alpha \mathrm{e}^{-\mathrm{i} k_{0} x}
$$

where the coefficient $\alpha$ depends on $\omega$. If the tank length is $L$ and the second wavemaker is stationary, perfect reflection will occur at $x=L$ with the reflected wave system represented by the potential

$$
\phi_{r}(x, 0) \simeq \xi_{1} \alpha \mathrm{e}^{-\mathrm{i} k_{0}(2 L-x)} .
$$

The sum of (12) and (13) is a standing wave with zero normal velocity on the wall $x=L$.

The second wavemaker, located at $x=L$ and oscillating with amplitude $\xi_{2}$, radiates a wave system moving toward $x=0$ with the form

$$
\xi_{2} \phi_{2}(x, 0) \simeq \xi_{2} \alpha \mathrm{e}^{-\mathrm{i} k_{0}(L-x)} .
$$

This will cancel the reflected system (13) if

$$
\xi_{2}=-\xi_{1} \mathrm{e}^{-\mathrm{i} k_{0} L} .
$$


This construction, based on the cancelation of the reflected wave system, gives the same result for the absorber amplitude as in the kinematic derivation represented in the more general three-dimensional case by (10). Note that the amplitude $\xi_{j}$ is defined in the positive sense with reference to the normal vector on the wavemaker element. Thus the minus sign in (15) is consistent with (10).

Reflections from the wavemaker at $x=0$ are ignored in the above analysis. To complete the solution it is necessary to add reflected waves from the periodic image boundaries at $x= \pm n L$ for all integer values of $n$. This leads to a modified steady state, with the sum of (12-14) replaced by

$$
\phi(x, 0) \simeq \frac{-\mathrm{i} \alpha}{\sin k_{0} L}\left[\xi_{1} \cos k_{0}(L-x)+\xi_{2} \cos k_{0} x\right],
$$

but the relation (15) for the optimum absorber amplitude is unchanged. In general the solution (16) is singular at the resonant 'sloshing' modes of the basin where $k_{0} L=n \pi$, but (16) reduces to (12) when (15) is substituted for the absorber amplitude. Thus the absorber prevents the occurrence of resonant modes.

Using (6), the pressure force acting on each wavemaker is given by

$$
\begin{gathered}
F_{1}=\omega^{2}\left(\xi_{1} A_{11}+\xi_{2} A_{12}\right)=\omega^{2} \xi_{1}\left(A_{11}-\mathrm{e}^{-\mathrm{i} k_{0} L} A_{12}\right), \\
F_{2}=\omega^{2}\left(\xi_{1} A_{12}+\xi_{2} A_{22}\right)=\omega^{2} \xi_{2}\left(A_{22}-\mathrm{e}^{\mathrm{i} k_{0} L} A_{21}\right) .
\end{gathered}
$$

The last factors in parenthesis can be interpreted as the 'effective' added mass and damping of each wavemaker by itself, when their motions are optimized according to (15). From symmetry it follows that $A_{11}=A_{22}$ and $A_{12}=A_{21}$. Thus the effective added mass

$$
a=A_{11}-A_{12} \cos k_{0} L
$$

is the same for both wavemakers. The effective damping coefficients are $\pm b$ where the upper sign applies to the wave generator at $x=0$, the lower sign to the absorber at $x=L$, and

$$
b / \omega=-A_{12} \sin k_{0} L .
$$

It is logical to expect that the damping is positive for the generator and negative for the absorber. This can be confirmed from the analysis in Appendix A, using (59) and neglecting the infinite series which is exponentially small for physically relevant values of the length $L$.

Since there is only one absorber, the equation of motion (11) for dynamic control is

$$
\xi_{2}\left(A_{22}+m-\mathrm{i} d / \omega\right)=-A_{12} \xi_{1} .
$$

Optimum values for the inertia and damping coefficients can be derived by substituting (15) in (21). Thus

$$
m=-a \quad \text { and } \quad d=b,
$$

where (19) and (20) have been used. These relations are noted by Naito [3] and attributed to Bessho [8]. From the physical viewpoint, the external inertia force should cancel the added mass (or more generally, should be evaluated with a corresponding stiffness coefficient to achieve resonance), and the optimum external damping is equal to the hydrodynamic damping. Similar relations are well known in the field of wave-power conversion, as reviewed by Evans [9]. 


\section{Generation and absorption of oblique waves}

The analysis in Section 3 can be extended for oblique wave generation and absorption by 'snake' wavemakers which extend to $y= \pm \infty$, with the horizontal displacement of each wavemaker proportional to $\mathrm{e}^{-\mathrm{i} k_{0} y \sin \beta}$. Neglecting the evanescent near-field motion, the potential on the free surface due to the wavemaker at $x=0$ is

$$
\xi_{1} \phi_{1}(x, 0) \mathrm{e}^{-\mathrm{i} k_{0} y \sin \beta} \simeq \xi_{1} \alpha \mathrm{e}^{-\mathrm{i} k_{0}(x \cos \beta+y \sin \beta)} .
$$

In the case where the fluid domain extends to $x=\infty$, analogous to the two-dimensional wavemaker in a semi-infinite domain, the differential hydrodynamic pressure force acting on the wavemaker can be expressed in the form

$$
F_{1}^{\prime}=\left(\omega^{2} A^{\prime}-\mathrm{i} \omega B^{\prime}\right) \xi_{1} \mathrm{e}^{-\mathrm{i} k_{0} y \sin \beta},
$$

where the added mass $A^{\prime}$ and damping $B^{\prime}$ depend on $\beta$. These coefficients are derived in Appendix B. The dependence of $A^{\prime}$ on $\beta$ is illustrated in Figure 1 . The dependence of $B^{\prime}$ on $\beta$ is given by the simple relation

$$
B^{\prime}(\beta)=B^{\prime}(0) \csc \beta=B \csc \beta,
$$

where $B$ is the two-dimensional damping coefficient (52). Thus the damping in oblique waves is greater than in two dimensions.

If the fluid domain is bounded by a wall at $x=L$, the reflection of the wave system $(23)$ can be canceled by an absorber at $x=L$ if

$$
\xi_{2}=-\xi_{1} \mathrm{e}^{-\mathrm{i} k_{0} L \cos \beta}
$$

In this case $\phi_{1}$ and $\phi_{2}$ are real, and the differential force on each wavemaker is given by

$$
\begin{aligned}
& F_{1}^{\prime}=\omega^{2}\left(\xi_{1} A_{11}^{\prime}+\xi_{2} A_{12}^{\prime}\right) \mathrm{e}^{-\mathrm{i} k_{0} y \sin \beta}=\omega^{2} \xi_{1}\left(A_{11}^{\prime}-\mathrm{e}^{-\mathrm{i} k_{0} L \cos \beta} A_{12}^{\prime}\right) \mathrm{e}^{-\mathrm{i} k_{0} y \sin \beta} \\
& F_{2}^{\prime}=\omega^{2}\left(\xi_{1} A_{12}^{\prime}+\xi_{2} A_{22}^{\prime}\right) \mathrm{e}^{-\mathrm{i} k_{0} y \sin \beta}=\omega^{2} \xi_{2}\left(A_{22}^{\prime}-\mathrm{e}^{\mathrm{i} k_{0} L \cos \beta} A_{21}^{\prime}\right) \mathrm{e}^{-\mathrm{i} k_{0} y \sin \beta} .
\end{aligned}
$$

Thus the effective added mass and damping are

$$
a^{\prime}=A_{11}^{\prime}-A_{12}^{\prime} \cos \left(k_{0} L \cos \beta\right)
$$

and

$$
b^{\prime} / \omega=-A_{12}^{\prime} \sin \left(k_{0} L \cos \beta\right) .
$$

Neglecting exponentially small terms in (66) and (67), it follows from the analysis in Appendix $\mathrm{B}$ that (29) and (30) are equal to the coefficients $A^{\prime}$ and $B^{\prime} / \omega$ defined by (64) and (65).

If the equation of motion for dynamic absorber control is

$$
\xi_{2}\left(A_{22}^{\prime}+m-\mathrm{i} d / \omega\right)=-A_{12}^{\prime} \xi_{1},
$$

optimum values of the inertia and damping can be derived by substituting (26) in (31). Thus

$$
m=-a^{\prime} \text { and } \quad d=b^{\prime},
$$

as in (22), and optimum dynamic control can be achieved in oblique waves by using the corresponding added-mass and damping coefficients $A^{\prime}$ and $B^{\prime}$ in place of the two-dimensional coefficients $A$ and $B$. 


\section{Three-dimensional wavemakers and basins}

The three-dimensional case is treated numerically, using the radiation-diffraction code WAMIT. Two examples are considered, a square basin $16 \mathrm{~m}$ by $16 \mathrm{~m}$ by $2 \mathrm{~m}$ depth, and a circular basin of radius $10 \mathrm{~m}$ and depth $2 \mathrm{~m}$. Hinged wavemakers are distributed uniformly around the periphery, with the hinges at the bottom of the basin. For the square basin the generating wavemakers are on two adjacent sides and the absorbing wavemakers are on the two opposite sides. For the circular basin the generating and absorbing wavemakers occupy opposite semi-circular arcs. In Figures 2-4 the generating elements are shown in black and the absorbers in red. Results are shown for the square basin at the incidence angles $\beta=0$ and $\beta=30^{\circ}$, relative to the $+x$-axis. For the circular basin the incidence angle is $\beta=90^{\circ}$.

The results presented here are for a period of 2 seconds, corresponding to a wavelength of $6.05 \mathrm{~m}$. Figures 2-4 show contour plots of the wave amplitude over the free surface, excluding a $1 \mathrm{~m}$ strip adjacent to the wavemakers. For progressive waves of the form (8) the amplitude is spatially constant. Reflections and other imperfections in the incident wave system are indicated by fluctuations of the amplitude. The magnitude of the fluctuations is measured in each plot by the standard deviation $\sigma$, defined as the square-root of the variance over the computational domain displayed in the figures and normalized by the mean value. Similar results are shown in [10] for basins with a depth of $1 \mathrm{~m}$. The reflection coefficient, defined as the difference between the maximum and minimum elevations divided by their sum, is 2-3 times larger than $\sigma$, but in most cases the largest fluctuations are near the outer boundary of the computational domain and the reflection coefficient near the center of the basin is smaller than this estimate would indicate.

Four separate contour plots are included in each figure. The upper left plots (a) show the standing waves which are generated when the absorbers are stationary. The upper right plots (b) show the wave amplitude with kinematic absorption. The lower left plots (c) show the results with dynamic absorption using the two-dimensional added mass and damping to evaluate the absorber coefficients $m$ and $d$ in accordance with (22). The lower right plots $(d)$ show the results with dynamic absorption using the oblique-wave added mass and damping to evaluate the absorber coefficients $m$ and $d$ in accordance with (32), where $\beta$ is defined as the local incidence angle relative to each absorber.

Figure 2 shows the wave amplitudes in the square basin for $\beta=0$. Two-dimensional standing waves are present in Figure 2(a). In Figures 2(b) and 2(d) the absorption is almost perfect, with $\sigma=0.004$. The results in Figure 2(c) show the limitation of using dynamic control with two-dimensional added mass and damping for all of the absorbers; in this case with $\beta=0$ the absorbers on the lower right wall should be stationary, as in Figure 2(b), but instead they react to the pressure of the passing wave system to extract energy and distort the waves. In Figure 2(d) this does not occur since the local angle of incidence is $90^{\circ}$, resulting in 'infinite' damping of the controller according to (32) and (25). (In the post-processor used to compute these results a finite upper bound $\csc \beta \leq 10^{4}$ is employed to evaluate the damping.)

Figure 3 shows the corresponding results For $\beta=30^{\circ}$. The standing-wave system in Figure 3(a) is three-dimensional, with substantial amplitude. This can be attributed to the proximity of a resonant mode at 1.98 seconds with five longitudinal nodes and two transverse nodes, not unlike the amplitude in Figure 3(a). The results using dynamic absorption with twodimensional coefficients, in Figure 3(c), are better than the corresponding results in Figure 2(c), but substantial reflections are still present. The results in Figures 3(b) and 3(d) are clearly superior. It is surprising to note that the standard deviation in $3(\mathrm{~d})$ is smaller than $3(\mathrm{~b})$, and this implies that the values of $\sigma$ are not accurate in the third decimal place.

The results for the circular basin in Figure 4 are similar to the results for the square basin 


\begin{tabular}{cccc}
$\beta$ & $64 \times 1 \mathrm{~m}$ & $128 \times 0.5 \mathrm{~m}$ & $256 \times 0.25 \mathrm{~m}$ \\
\hline 0 & 0.0036 & 0.0036 & 0.0036 \\
$22.5^{\circ}$ & 0.0324 & 0.0080 & 0.0032 \\
$45^{\circ}$ & 0.0505 & 0.0127 & 0.0039
\end{tabular}

Table 1: Effect of the wavemaker width on the variance $\sigma$ at different incidence angles, for 64, 128, and 256 wavemakers in the square basin.

in oblique waves, with similar conclusions regarding the different absorber schemes.

For the results shown in Figures 2-4 a total of 128 wavemakers are used, with a width of $0.5 \mathrm{~m}$. Table 1 shows the effect on the variance $\sigma$ using different widths of $1.0 \mathrm{~m}, 0.5 \mathrm{~m}$, and $0.25 \mathrm{~m}$, with kinematic absorbers. The uniformity of oblique waves is improved by reducing the width of the wavemakers, as expected.

\section{Absorption of radiated and scattered waves}

Bodies which are present in the basin will radiate waves, due to their motions, and also scatter the incident waves generated by wavemakers. The radiated and scattered waves propagate outward toward the sides of the basin. It is necessary to absorb these waves in addition to the incident wave field. Indeed, this more general requirement is the principal reason for using dynamic absorbers.

In order to illustrate this type of problem we consider the case of a floating hemisphere of radius $1 \mathrm{~m}$ at the center of the basin. The simplest radiation problem to consider is heaving motion of the hemisphere in the circular basin, since the motion is axisymmetric and the absorbing wavemakers can be assumed to have the same amplitude and phase. Two alternative approaches are used to optimize the wavemaker motion in this case.

First we consider the two standing-wave systems generated by the separate motions of the hemisphere and wavemakers. Their amplitudes can be represented in the form

$$
\xi_{i} \eta_{i}=\xi_{i}\left(C_{i} \mathrm{e}^{\mathrm{i} k_{0} r}+C_{i}^{*} \mathrm{e}^{-\mathrm{i} k_{0} r}\right) / \sqrt{k_{0} r},
$$

where $i=1$ denotes the hemisphere, $i=2$ denotes the wavemaker, $C_{i}$ is a complex coefficient and $C_{i}^{*}$ denotes the conjugate. The first and second terms in parentheses represent inward and outward propagating ring waves, respectively. The inward propagating waves are canceled if

$$
\xi_{2} / \xi_{1}=-\left(C_{1} / C_{2}\right) .
$$

This is the optimum condition for the absorber, analogous to (15) in two dimensions. In order to evaluate $\xi_{2} / \xi_{1}$, computations are made of $\eta_{1}$ and $\eta_{2}$ along a radial line between $r=1.5 \mathrm{~m}$ and $r=9.5 \mathrm{~m}$ in steps of $0.1 \mathrm{~m}$. The coefficients $C_{i}$ are evaluated by Fourier integration of this data. Figure 5 shows the amplitudes of the two standing-wave components (33) for several wave periods, and also the radiated wave $\eta_{1}-\left(C_{1} / C_{2}\right) \eta_{2}$. Using (6), the force acting on the hemisphere in the latter condition is

$$
F_{1}=\omega^{2} \xi_{1}\left[A_{11}-\left(C_{1} / C_{2}\right) A_{12}\right] .
$$

The added mass and damping are given by the real and imaginary parts of the factor in square brackets.

As an alternative procedure, analogous to (21), the equation of motion for the absorber amplitude is solved using the two-dimensional added-mass and damping coefficients. This 
avoids measurement and analysis of the free-surface elevation, and is simpler to employ in the experimental context. Figure 6 shows the added-mass and damping coefficients of the hemisphere, comparing the results from the two approaches with computations for the same body in open water. The results based on the first approach are practically identical to the open-water results. The second approach introduces a small oscillatory error for longer wave periods, which may be explained by the fact that the two-dimensional added-mass and damping coefficients are affected by the curvature of the wavemaker to a greater extent in longer waves.

Next we consider the diffraction problem where the hemisphere is fixed and plane progressive waves are generated by the wavemakers with $\beta=0$. Two separate sets of computations are performed, without and with the hemisphere present in the basin. The first computation is a 'calibration' of the basin and wavemakers, where the amplitude $\tilde{\xi}_{j}$ of the wavemakers is defined by (10), as in Figures 2(b) and 4(b). The resulting incident wave amplitude $\tilde{A}$ is computed at the center of the basin, and the added-mass coefficients $\tilde{A}_{i j}$ are evaluated for the wavemakers.

In the second computation, with the hemisphere present in the basin, the added-mass matrix $A_{i j}$ is evaluated including the hydrodynamic interactions between the hemisphere and wavemakers. In general there are six additional modes, corresponding to the rigid-body motions of the hemisphere, but it is only necessary to account for surge and heave, denoted by the subscripts $x$ and $z$ respectively. The amplitudes $\xi_{j}$ for the wavemakers, defined as the solution of the linear system (11), can be expressed in the form

$$
\xi_{j}=\tilde{\xi}_{j}+\delta_{j}
$$

where $\delta_{j}$ is the correction to account for scattering. Since $\tilde{\xi}_{j}$ is the solution of (11) with $A_{i j}$ replaced by $\tilde{A}_{i j}$, it follows that

$$
\sum_{j=1}^{N} A_{i j} \delta_{j}+\left(m_{i}-\mathrm{i} d_{i} / \omega\right) \delta_{i}=-\sum_{j=1}^{N}\left(A_{i j}-\tilde{A}_{i j}\right) \tilde{\xi}_{j}, \quad(i=1,2, \ldots, N) .
$$

Note that this linear system is applied for all $N$ wavemakers, and the generators of the incident wave also serve to absorb back-scattered waves from the body.

The principal advantage of solving (37) for $\delta_{j}$ is that the kinematic amplitude (10) is used for the incident waves, and any approximations associated with the absorbers are confined to the component $\delta_{j}$. In the following evaluation of the exciting force, the inertia and damping coefficients $m_{i}$ and $d_{i}$ are replaced by the corresponding constant values for a two-dimensional wavemaker.

The added-mass coefficients $A_{x j}, A_{z j} \quad(j=1,2, \ldots, N)$ represent cross-coupling between the sphere and wavemakers. These coefficients can be used with the solution of (37) to evaluate the exciting force components

$$
\left(\begin{array}{c}
F_{x} \\
F_{z}
\end{array}\right)=\omega^{2} \sum_{j=1}^{N}\left(\begin{array}{c}
A_{x j} \\
A_{z j}
\end{array}\right) \xi_{j}
$$

The values of $\left|F_{x}\right|$ and $\left|F_{z}\right|$ are shown in Figure 7 for the square and circular basins, and compared with the exciting forces in open water. The maximum absolute difference between the open-water computations and basins is about 0.03. For the phase angles, the maximum difference is about $4^{\circ}$. The most noticeable differences in Figure 7 are for the circular basin, in longer wavelengths; these appear to be similar to the larger differences in Figure 6, which are attributed to the effects of curvature in longer wavelengths. Small differences are apparent also for the surge force at periods between 1.0 and 1.1 seconds, which may be attributed to discretization errors at the shortest wavelengths. 
The same computations have been performed for the square basin using oblique added-mass and damping coefficients, based on the angle of incidence from the center of the basin (varying between $0^{\circ}$ at the center of each side and $45^{\circ}$ at the corners). The exciting forces computed in this manner are practically the same as those based on the two-dimensional coefficients.

Additional computations, not shown here, have been performed with the exciting forces evaluated from (38) using the amplitudes $\tilde{\xi}_{j}$, without correcting for the effect of scattering. These results are highly oscillatory in both basins, compared to the results in Figure 7, confirming that while the scattered waves may be small compared to the incident waves, large standing waves can result from their reflections especially in the vicinity of wave periods where resonant modes exist.

\section{Two-dimensional wavemakers in the time-domain}

Transient effects are significant in physical experiments, even when the objective is to produce a monochromatic wave system. To illustrate these effects we consider a two-dimensional wavemaker at $x=0$, starting from rest at time $t=0$ and moving with normal velocity $U(t) f(z)$. The velocity potential can be represented by a distribution of sources over the surface of the wavemaker, with the source density equal to the normal velocity. To simplify the analysis it will be assumed that the fluid depth is infinite, and thus the appropriate source potential is given by Wehausen \& Laitone ([11], eq. 13.54). The free-surface elevation is represented by the convolution integral

$$
\zeta(x, t)=\int_{0}^{t} U(\tau) k(x, t-\tau) d \tau
$$

where the impulse-response function is

$$
k(x, t)=-\frac{1}{\pi} \int_{0}^{\infty} \cos \kappa x \cos \sqrt{g \kappa} t \int f(z) \mathrm{e}^{\kappa z} d z d \kappa .
$$

A hinged wavemaker is considered, as in the preceding Sections, with $f(z)$ defined by (3) and the hinge axis $2 \mathrm{~m}$ below the free surface. After evaluating the integral with respect to $z$ analytically, the remaining integral with respect to $\kappa$ is evaluated numerically, as explained in Section 8 . Figure 8 shows plots of this function at six successive time steps. These results are similar to the waves due to a concentrated impulse on the free surface (cf. Lamb [12], §§238-239), usually referred to as the Cauchy-Poisson problem. The longer waves propagate with relatively large velocity, and shorter waves follow behind. Unlike the solution for a concentrated impulse, the shorter waves in Figure 8 diminish in magnitude due to the vertical distribution of the sources on the wavemaker.

Hereafter it is assumed that the wavemaker velocity $U(t)=\sin \omega t$ for $t>0$, with the wave period $2 \pi / \omega=2$ seconds. The generated waves are shown for a sequence of time steps in Figure 9. The dashed lines in Figure 9 confirm that the wave front moves with the group velocity and the individual wave crests move with the phase velocity. The waves behind the front quickly approach their limiting sinusoidal form. Figure 6.10 in [13] shows a similar sequence of waves based on photographs in a small physical wave tank.

If the wavemaker is at one end of a basin, with an absorbing wavemaker at the opposite end, the synthesis described in Section 3 can be replicated in the time domain. For illustration a basin of length $L=40 \mathrm{~m}$ is used, with the generating wavemaker at $x=0$ and the absorber at $x=40 \mathrm{~m}$. An image wavemaker at $x=80 \mathrm{~m}$ is used to simulate the reflected wave, with the same velocity $U(t)$ as the generator. The absorber velocity is $-U\left(t-k_{0} L / \omega\right)$, where the time lag corresponds to the phase lag $-\mathrm{i} k_{0} L$ in (15). The superposition of these three wave systems 
is shown in Figure 10. By following the crests along the lower dashed line it can be confirmed that a steady propagating wave system is achieved, but only after a substantial time. Prior to this partial standing waves are present. These are due primarily to starting the wave absorber too soon, as indicated by the waves that exist in Figure 10 ahead of the original front (above the upper dashed line near the right end). This can be anticipated since the phase lag $-\mathrm{i} k_{0} L$ corresponds to a time delay propagating with the phase velocity instead of the group velocity.

As an alternative scheme the absorber velocity is assumed to be proportional to the freesurface elevation $\zeta_{I}$ due to the wave generator, as measured at the absorber. Thus the contribution by the absorber is

$$
\zeta_{A}(x, t)=C \int_{0}^{t} \zeta_{I}(L, \tau) k(L-x, t-\tau) d \tau
$$

Here the coefficient $C$ is analogous to the transfer function between $U$ and $\zeta_{I}$ in the frequency domain. The result of using this absorber algorithm is shown in Figure 11. It is clearly superior to Figure 10, with close approximation to a steady propagating wave system shortly after the incident-wave front reaches the absorber. In addition to delaying the absorber response until the waves arrive, this algorithm also accounts for the gradual build-up of the wave system at the front. Since the wave elevation and pressure are closely correlated, it is likely that similar performance can be achieved with $\zeta_{I}$ replaced in (41) by the pressure force acting on the absorber. This would give a time-domain representation of dynamic absorption.

\section{Computational details}

The numerical results shown in Figures 2-7 have been obtained using the radiation/diffraction program WAMIT. This program is intended primarily for the analysis of wave interactions with bodies in open water, but it can be used for internal fluid domains with a free surface, such as partially-filled tanks and wave basins. The principal requirement is that the submerged surface of the basin, as well as the body, is defined in the geometry input to the program. (For a basin with constant depth, as in the cases considered here, it is not necessary to include the bottom as part of the input geometry since the boundary condition on the bottom is satisfied by the Green function.)

Each component $\phi_{j}$ is evaluated as the solution of a discretized integral equation over the domain including the wavemakers and body. The motion of each wavemaker is represented by a generalized mode, with the normal velocity assigned in a special subroutine as a function of the vertical position on the wavemaker. The higher-order method of solution is used, with the unknown potentials represented by B-splines. The geometry of the basins and hemisphere are represented analytically, without approximations. Each wavemaker is represented by a separate patch, with subdivision used to achieve converged solutions. Further details can be found in [14]. Special post-processing utilities are used to convert the standard outputs from WAMIT to the results presented here.

In a closed basin each component $\phi_{j}$ should be real, as noted in Section 2. Since the exterior Green function used in the program is complex, one test of the numerical accuracy of the solution in a closed basin is to compare the magnitudes of the real and imaginary parts. Typically the computed ratios between the damping and added-mass coefficients are on the order of $10^{-3}$ or smaller. The corresponding ratios for the wave amplitudes are on the order of $10^{-5}$ or smaller.

The two-dimensional time-domain computations described in Section 7 have been performed with a program which evaluates the impulse-response function and convolution integrals by 
numerical quadrature. The impulse-response function (40) is evaluated as the sum of two separate integrals involving the sum and difference of the trigonometric arguments. These semi-infinite integrals are replaced by infinite series of finite integrals, defined such that the trigonometric arguments change by $2 \pi$ in each interval (except for one interval where the point of stationary phase occurs). Adaptive Romberg quadratures are used in each interval, with a convergence tolerance of $10^{-5}$, and the summations are continued until the last ten terms in the series are smaller than $10^{-6}$. The convolution integrals (39) and (41) are evaluated by the trapezoidal rule with time steps equal to 0.03 . This value of the time step gives converged results in Figures 9-11, within graphical accuracy.

\section{Discussion and Conclusions}

The generation and absorption of waves in a closed basin has been analyzed within the framework of linear potential theory. Wavemakers situated in the sides of the basin are used for both generating and absorbing the waves. Specific results are presented for square and circular basins, with wavemakers which are hinged at the bottom of the basin. Other geometrical configurations can be analyzed in a similar manner.

In a closed basin it is essential to absorb the incident waves generated by the wavemakers, as well as radiated and scattered waves from bodies within the basin. Two types of controls are considered for the absorbers, referred to as 'kinematic' and 'dynamic'. In the kinematic case all of the wavemaker elements oscillate with prescribed amplitudes of motion, and with appropriate phases, to coincide with the normal velocity of a progressive wave at the center of each wavemaker. If the wavemakers are sufficiently small, compared to the wavelength, this simple approach ensures that both the wave generation and absorption will be effective provided there are no bodies or other sources of radiation or scattering within the basin.

To provide for more general wave absorption, including radiation or diffraction from bodies, a dynamic scheme is introduced. Each absorbing element responds to the local pressure force from the incident waves, with an external linear controller which is represented by inertial and damping forces. Solution of the coupled equations of motion gives the amplitude and phase for each absorber. Optimum external control is achieved if the inertia force is equal to the negative added mass and the damping force is equal to the wave damping, based on Havelock's wavemaker theory. These optimum coefficients are evaluated for normal incidence based on two-dimensional theory in Appendix A, and for oblique incidence by a generalization of the same theory in Appendix B.

Computations are performed in the frequency domain using the radiation/diffraction code WAMIT. Contour plots of the wave amplitude throughout the basin are shown comparing the results without absorbers, with kinematic absorbers, and with dynamic absorbers. The quality of the absorbers is measured by the variance of the wave amplitude. Without absorbers there are severe standing waves due to reflection from the walls opposite to the wave generators, and from the generators themselves. With kinematic absorbers the variance is very small, and there is practically no reflection. The effectiveness of dynamic absorbers depends on the assumptions used to derive the added-mass and damping coefficients. For incident wave absorption in a basin without radiation and scattering from a body, dynamic absorbers are as effective as kinematic absorbers when oblique added-mass and damping coefficients are used to account for the local incident angle at the absorbers. The use of two-dimensional added-mass and damping coefficients is less satisfactory.

In order to study the effects of radiation and scattering, computations are performed with a floating hemisphere in the center of the basin. Two specific applications are considered, first to 
evaluate the added mass and damping due to heaving motion of the hemisphere in a circular basin, and then to evaluate the exciting force in incident waves for both the square and circular basins. In the heaving problem the motion is axisymmetric, and the absorbing wavemakers around the basin have the same amplitude and phase. The absorber is optimized first by analysis of the free-surface elevation in the basin, and then by a dynamic approach. Both give good approximations for the added mass and damping of the hemisphere in open water, but the dynamic approach shows small oscillatory errors in longer wavelengths which may be attributed to the use of two-dimensional theory for the determination of absorber control.

The evaluation of the exciting forces on the hemisphere is more challenging, since the threedimensional scattered wave field is superposed on the incident wave system and both must be absorbed to achieve satisfactory results. The method adopted here is to use kinematic absorption for the incident waves, and dynamic absorption for the difference in the wave field due to scattering. This gives good results for the exciting forces in both the square and circular basins, based on comparisons with separate computations in open water. Similarly good performance can be expected if dynamic absorbers are used for both the incident and scattered waves, provided their angle of incidence is measured and accounted for using oblique added-mass and damping coefficients.

The two-dimensional time-domain analysis in Section 8 illustrates some transient phenomena that are relevant to absorber control. Comparison of the results shown in Figures 10 and 11 suggests the utility of wavemaker control based on measuring the instantaneous free-surface elevation at the absorber. Similar results are expected using the exciting force in place of the elevation. In physical experiments it may be difficult to separate the contribution due to the incident waves from the total elevation or force including the absorber's own motion. Theoretical and numerical analysis may be useful in this context.

The dynamic absorber control is frequency-dependent. This complicates applications in the time domain, as discussed by Maisondieu \& Clément [15] and also by Naito [3]. Thus the present work may represent an upper bound on the performance of wave absorbers. 


\section{References}

[1] Milgram, J. 'Active water-wave absorbers,' J. Fluid Mech. 43, 845-859, 1970.

[2] Salter, S.H. 'Absorbing wave-makers and wide tanks,' Proc. Directional Wave Spectra Applications, Berkeley, 1981.

[3] Naito, S. 'Wave generation and absorption in wave basins: theory and application,' J. ISOPE, 16, 2, 81-89, 2006.

[4] Skourup, J. \& Bingham, H.B., 'Active absorption of radiated waves in a 3D boundary element model,' 11th IWWWFB, Hamburg, Germany, 1996. (www.iwwwfb.org)

[5] Grilli, S.T., Dias, F., Guyenne, P., Fochesato, C. and Enet, F., 'Progress in fully nonlinear potential flow modeling Of 3D extreme ocean waves,' in Advances in Numerical Simulation of Nonlinear Water Waves, World Scientific Publishing, 2008.

[6] O’Dea, J.F. and Newman, J.N., 'Numerical studies of directional wavemaker performance,' 28th American Towing Tank Conference, Ann Arbor, Michigan 2007.

[7] Havelock, T.H. 'Forced surface waves on water,' Phil. Mag. 8, 569-576, 1929.

[8] Bessho, M. 'Feasibility study of a floating-type wave absorber,' Proc. 34th Japan Towing Tank Conf, 1973 (in Japanese).

[9] Evans, D.V. 'Power from water waves,' Ann. Rev. Fluid Mech. 13, 157-187, 1981.

[10] Newman, J.N., 'Linear analysis of wave basins and absorbers,' 23rd IWWWFB, Jeju, Korea, 2008. (www.iwwwfb.org)

[11] Wehausen, J. V. \& Laitone, E. V., 'Surface Waves,' In Encyclopedia of Physics, Vol. IX, pp. 446-778, Springer Verlag, 1960 (www.coe. berkeley.edu/SurfaceWaves/)

[12] Lamb, H., 'Hydrodynamics,' Cambridge University Press, 6th ed., 1932. Reprinted by Dover Publications.

[13] Newman, J.N., 'Marine Hydrodynamics,' MIT Press, 1977.

[14] WAMIT, Inc, 'User Manual,' 2006. (www .wamit.com.)

[15] Maisondieu, C. \& Clément, A., 'A realizable force feedback-feedforward control loop for a piston wave absorber,' 8th IWWWFB, St John's, Newfoundland, 1993. (www.iwwwfb.org) 


\section{Appendix A - Solution for two-dimensional wavemaker}

A fluid of constant depth $h$ is bounded on the left by a vertical wavemaker at $x=0$. The wavemaker oscillates with amplitude $f(z) \cos \omega t$, and normal velocity $-\omega f(z) \sin \omega t$. If the harmonic time dependence is represented by the complex factor $\mathrm{e}^{\mathrm{i} \omega t}$, the velocity potential $\phi$ satisfies the boundary conditions

$$
\begin{gathered}
\frac{\partial \phi}{\partial x}=\mathrm{i} \omega f(z) \quad \text { on } x=0, \\
\frac{\partial \phi}{\partial z}=0 \quad \text { on } z=-h,
\end{gathered}
$$

and the free-surface condition (4). Following Havelock [7], the potential can be expressed in the general form

$$
\phi=C_{0} \cosh k_{0}(z+h) \mathrm{e}^{ \pm \mathrm{i} k_{0} x}+\sum_{n=1}^{\infty} C_{n} \cos k_{n}(z+h) \mathrm{e}^{ \pm k_{n} x} .
$$

Here $k_{0}$ is the positive real root of the dispersion relation (9) and $k_{n}$ denotes the positive imaginary roots. The eigenfunctions $\left(\cosh k_{0}(z+h), \cos k_{n}(z+h)\right)$ are complete and orthogonal in the domain $(-h \leq z \leq 0)$.

It is convenient to define the integrals

$$
\begin{gathered}
c_{0}=\int_{-h}^{0} f(z) \cosh k_{0}(z+h) d z=\left(k_{0} h \sinh k_{0} h-\cosh k_{0} h+1\right) /\left(k_{0}^{2} h\right), \\
c_{n}=\int_{-h}^{0} f(z) \cos k_{n}(z+h) d z=\left(k_{n} h \sin k_{n} h+\cos k_{n} h-1\right) /\left(k_{n}^{2} h\right),
\end{gathered}
$$

where the last results in (45) and (46) apply for a hinged wavemaker with $f(z)=(z+h) / h$.

For the usual case of a semi-infinite fluid domain $(0<x<\infty)$ a radiation condition is imposed with waves moving in the $+x$ direction. Thus

$$
\phi=C_{0} \cosh k_{0}(z+h) \mathrm{e}^{-\mathrm{i} k_{0} x}+\sum_{n=1}^{\infty} C_{n} \cos k_{n}(z+h) \mathrm{e}^{-k_{n} x},
$$

where

$$
\begin{aligned}
& C_{0}=\frac{-4 \omega c_{0}}{\sinh 2 k_{0} h+2 k_{0} h}, \\
& C_{n}=\frac{-4 \mathrm{i} \omega c_{n}}{\sin 2 k_{n} h+2 k_{n} h} .
\end{aligned}
$$

The added-mass and damping coefficients follow from the integral of the pressure force acting on the wavemaker,

$$
\omega^{2} A-\mathrm{i} \omega B=-\mathrm{i} \rho \omega \int_{-h}^{0} f(z) \phi(0, z) d z .
$$

Thus

$$
\begin{gathered}
A=4 \rho \sum_{n=1}^{\infty} \frac{c_{n}^{2}}{\sin 2 k_{n} h+2 k_{n} h}, \\
B=4 \rho \omega \frac{c_{0}^{2}}{\sinh 2 k_{0} h+2 k_{0} h} .
\end{gathered}
$$


More generally, if the hinge depth $h$ is less than the fluid depth $H$, equations (45) and (46) are replaced by

$$
\begin{gathered}
c_{0}=\int_{-h}^{0} f(z) \cosh k_{0}(z+H) d z=\left[\cosh k_{0}(H-h)\left(k_{0} h \sinh k_{0} h-\cosh k_{0} h+1\right)\right. \\
\left.+\sinh k_{0}(H-h)\left(k_{0} h \cosh k_{0} h-\sinh k_{0} h\right),\right] /\left(k_{0}^{2} h\right) \\
c_{n}=\int_{-h}^{0} f(z) \cos k_{n}(z+H) d z=\left[\cos k_{n}(H-h)\left(k_{n} h \sin k_{n} h+\cos k_{n} h-1\right)\right. \\
\left.\quad+\sin k_{n}(H-h)\left(k_{n} h \cos k_{n} h-\sin k_{n} h\right)\right] /\left(k_{n}^{2} h\right) .
\end{gathered}
$$

For a basin of finite length, with the fluid domain $(0<x<L)$, the radiation condition is replaced by the boundary condition $\phi_{x}=0$ on $x=L$ and the potential is

$$
\phi=C_{0} \cosh k_{0}(z+h) \cos \left(k_{0}(L-x)\right)+\sum_{n=1}^{\infty} C_{n} \cos k_{n}(z+h) \cosh \left(k_{n}(L-x)\right) \mathrm{e}^{-k_{n} L},
$$

where

$$
\begin{aligned}
C_{0} & =\frac{4 c_{0} \omega}{\left(\sinh 2 k_{0} h+2 k_{0} h\right) \sin k_{0} L}, \\
C_{n} & =\frac{-4 c_{n} \omega \mathrm{e}^{k_{n} L}}{\left(\sin 2 k_{n} h+2 k_{n} h\right) \sinh k_{n} L} .
\end{aligned}
$$

In this case there is no damping, and the added-mass coefficients defined in Section 3 are

$$
\begin{aligned}
& A_{11}=\rho \int_{-h}^{0} f(z) \phi(0, z) d z=-4 \rho \frac{c_{0}^{2} \cot k_{0} L}{\sinh 2 k_{0} h+2 k_{0} h}+4 \rho \sum_{n=1}^{\infty} \frac{c_{n}^{2} \operatorname{coth} k_{n} L}{\sin 2 k_{n} h+2 k_{n} h}, \\
& A_{12}=\rho \int_{-h}^{0} f(z) \phi(L, z) d z=-4 \rho \frac{c_{0}^{2} \csc k_{0} L}{\sinh 2 k_{0} h+2 k_{0} h}+4 \rho \sum_{n=1}^{\infty} \frac{c_{n}^{2} \operatorname{csch} k_{n} L}{\sin 2 k_{n} h+2 k_{n} h} .
\end{aligned}
$$

The coefficients $(58,59)$ are singular when $k_{0} L=n \pi$, but the effective added mass and damping $(19,20)$ are nonsingular. When $L / h>>1$ the differences between $(19,20)$ and $(51,52)$ are exponentially small.

\section{Appendix B - Solution for oblique waves}

The analysis in Appendix A can be extended for oblique wave generation and absorption by 'snake' wavemakers in the planes $x=0$ and $x=L$, with sinusoidal variation of the amplitude in the $y$-direction. Assuming the wavemakers extend to infinity in both directions, the velocity potential can be represented in the form $\phi(x, z) \mathrm{e}^{-\mathrm{i} k_{0} y \sin \beta}$. The boundary conditions (42) and (43) are unchanged, and (44) is replaced by

$$
\phi=C_{0}^{\prime} \cosh k_{0}(z+h) \mathrm{e}^{ \pm \mathrm{i} k_{0}^{\prime} x}+\sum_{n=1}^{\infty} C_{n}^{\prime} \cos k_{n}(z+h) \mathrm{e}^{ \pm k_{n}^{\prime} x}
$$

where, in order to satisfy the Laplace equation,

$$
k_{0}^{\prime}=k_{0} \cos \beta
$$


and

$$
k_{n}^{\prime}=\sqrt{k_{n}^{2}+k_{0}^{2} \sin ^{2} \beta} .
$$

For the case where the fluid domain extends to $x=\infty$ (47) is replaced by

$$
\phi=C_{0}^{\prime} \cosh k_{0}(z+h) \mathrm{e}^{-\mathrm{i} k_{0}^{\prime} x}+\sum_{n=1}^{\infty} C_{n}^{\prime} \cos k_{n}(z+h) \mathrm{e}^{-k_{n}^{\prime} x}
$$

where

$$
\begin{gathered}
C_{0}^{\prime}=C_{0} \csc \beta, \\
C_{n}^{\prime}=C_{n}\left(k_{n} / k_{n}^{\prime}\right) .
\end{gathered}
$$

and the coefficients $C_{0}$ and $C_{n}$ are given by (48) and (49). The added-mass and damping coefficients defined by (24) are

$$
\begin{aligned}
& A^{\prime}=4 \rho \sum_{n=1}^{\infty} \frac{c_{n}^{2}\left(k_{n} / k_{n}^{\prime}\right)}{\sin 2 k_{n} h+2 k_{n} h}, \\
& B^{\prime}=4 \rho \omega \frac{c_{0}^{2} \csc \beta}{\sinh 2 k_{0} h+2 k_{0} h} .
\end{aligned}
$$

Thus the damping coefficient for oblique-wave generation is simply proportional to csc $\beta$. This can be confirmed by noting from the $x$-derivative of (8) that the amplitude of the generated waves, for unit wavemaker amplitude, is proportional to $\csc \beta$. Thus the energy density is proportional to $\csc ^{2} \beta$. Since the component of the group velocity normal to a control surface $x=$ constant is proportional to $\cos \beta$, the rate of energy flux is proportional to $\csc \beta$.

For a basin of finite length the added-mass coefficients $(58,59)$ are replaced by

$$
\begin{aligned}
& A_{11}^{\prime}=\rho \int_{-h}^{0} f(z) \phi(0, z) d z=-4 \rho \frac{c_{0}^{2} \cot k_{0}^{\prime} L \csc \beta}{\sinh 2 k_{0} h+2 k_{0} h}+4 \rho \sum_{n=1}^{\infty} \frac{c_{n}^{2}\left(k_{n} / k_{n}^{\prime}\right) \operatorname{coth} k_{n}^{\prime} L}{\sin 2 k_{n} h+2 k_{n} h}, \\
& A_{12}^{\prime}=\rho \int_{-h}^{0} f(z) \phi(L, z) d z=-4 \rho \frac{c_{0}^{2} \csc k_{0}^{\prime} L \csc \beta}{\sinh 2 k_{0} h+2 k_{0} h}+4 \rho \sum_{n=1}^{\infty} \frac{c_{n}^{2}\left(k_{n} / k_{n}^{\prime}\right) \operatorname{csch} k_{n}^{\prime} L}{\sin 2 k_{n} h+2 k_{n} h} .
\end{aligned}
$$

As in the case of two dimensions, $\phi$ is real and the local force acting on the wavemakers is expressed completely by the added-mass coefficients. 


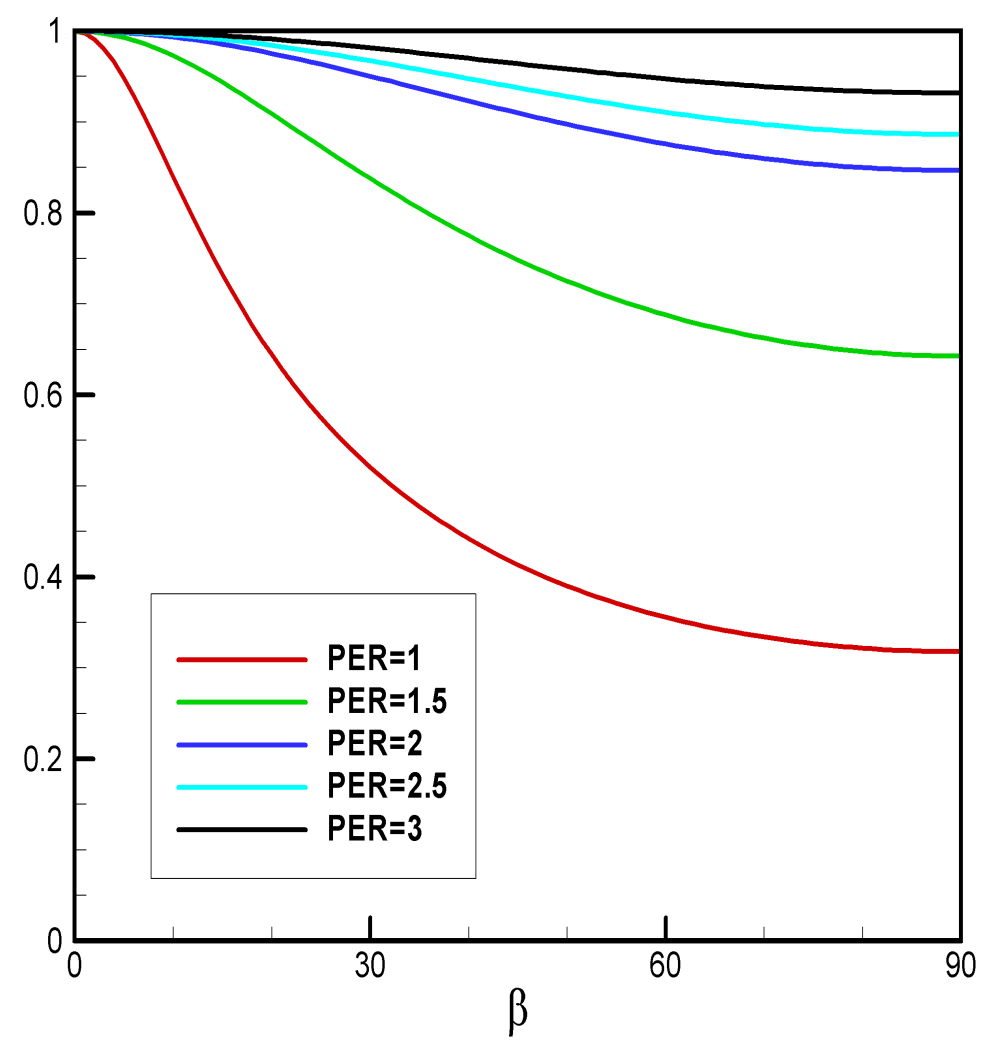

Figure 1: Effect of oblique incidence on the added mass of the 'snake wavemaker'. These results are based on (64), and normalized by the limiting value for $\beta=0$, which corresponds to the two-dimensional added mass (51). Wave periods (PER) are in seconds. 

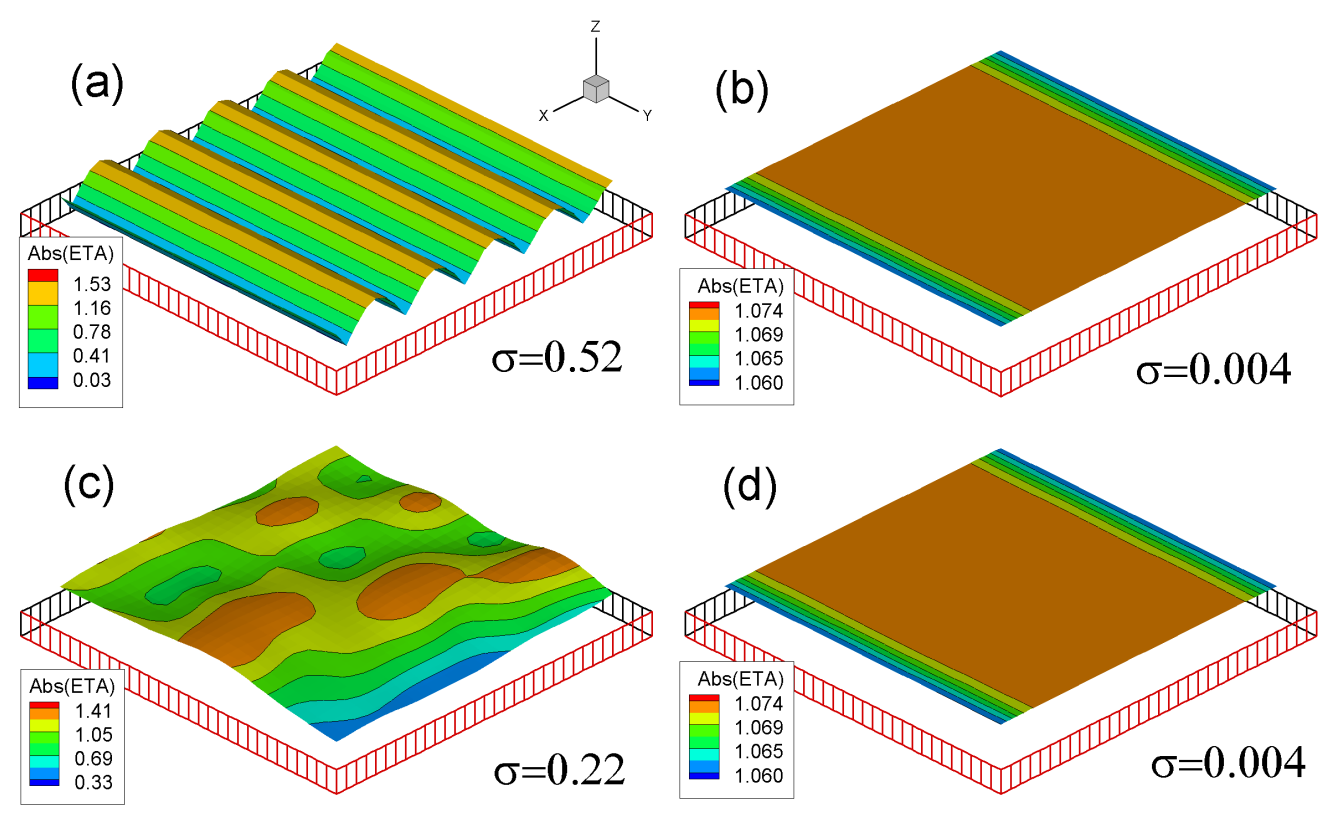

Figure 2: Amplitude of waves in the square basin with generators (black) and absorbers (red). The waves propagate in the $+x$-direction $(\beta=0)$. The upper left plot (a) shows the standing-wave system without absorption. The absorbers are (b) kinematic, (c) dynamic with 2D added-mass and damping forces, and (d) dynamic with oblique added-mass and damping forces. Note that different ranges of colors are used to represent the amplitude in each plot. The variance $\sigma$ indicates the magnitude of reflections and nonuniformity in the wave system.

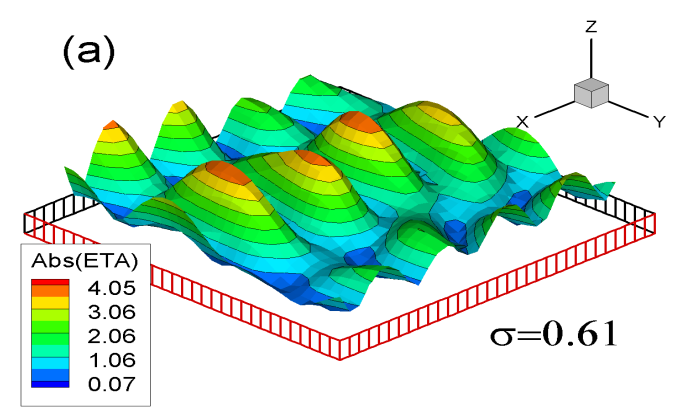

(c)

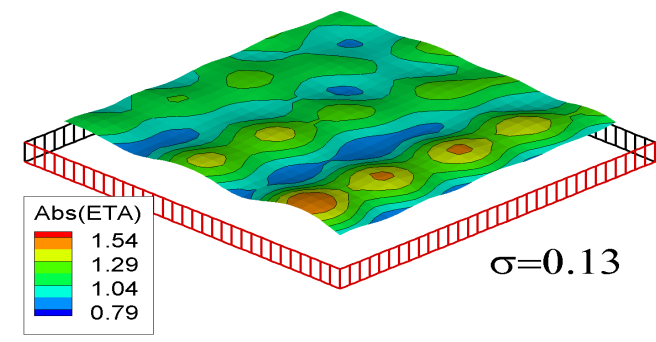

(b)

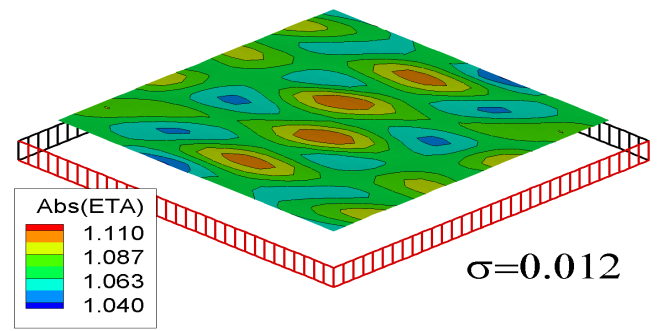

(d)

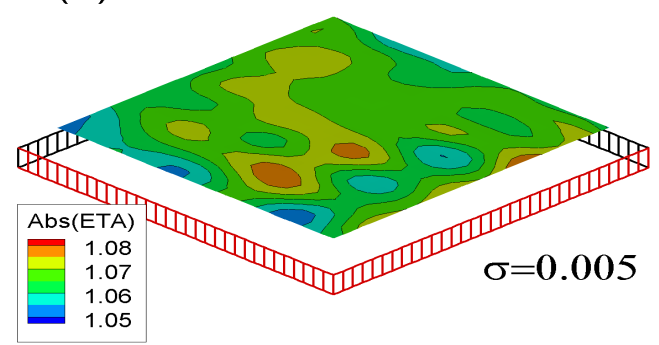

Figure 3: Amplitude of waves in the square basin with generators (black) and absorbers (red). The waves propagate in the direction $\left(\beta=30^{\circ}\right)$ relative to the $+x$-axis. The upper left plot (a) shows the standing-wave system without absorption. The absorbers are (b) kinematic, (c) dynamic with 2D added-mass and damping forces, and (d) dynamic with oblique added-mass and damping forces. Note that different ranges of colors are used to represent the amplitude in each plot. The variance $\sigma$ indicates the magnitude of reflections and nonuniformity in the wave system. 

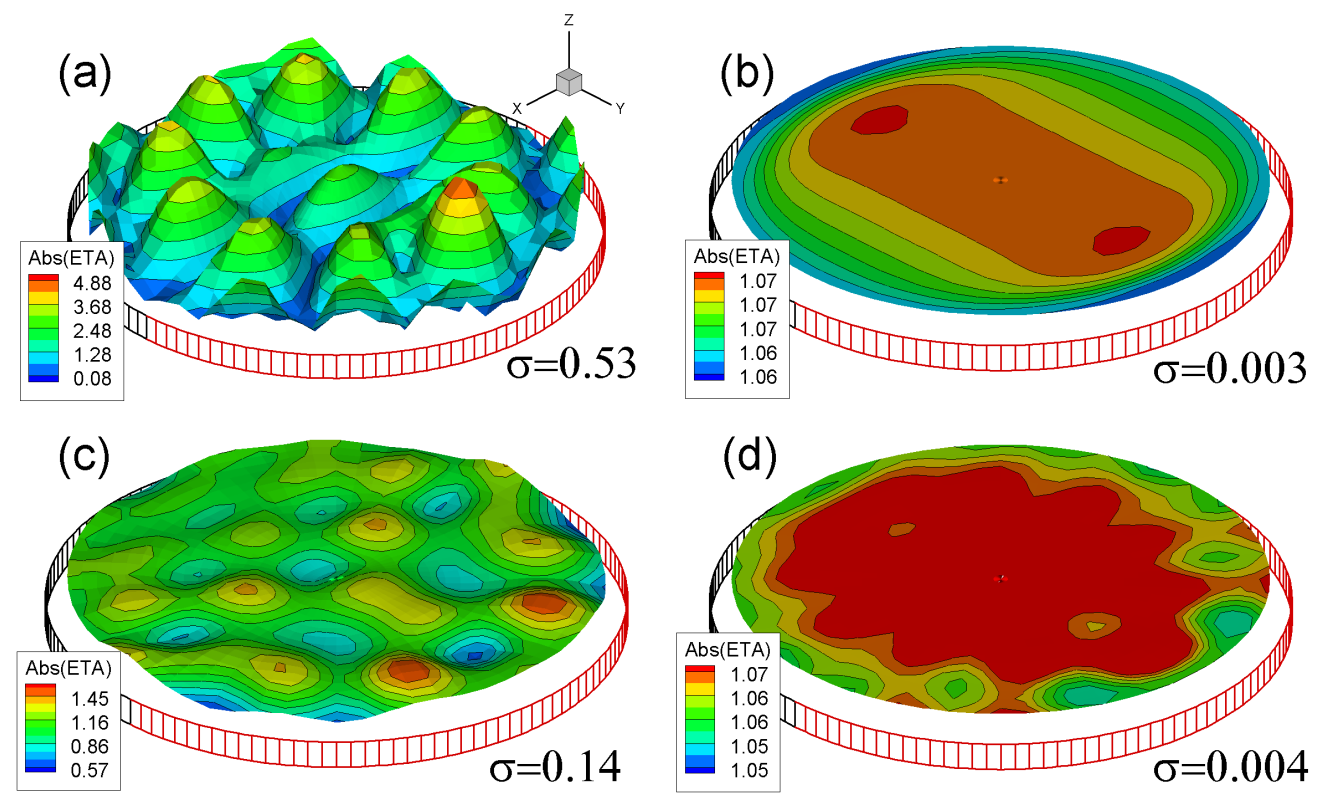

Figure 4: Amplitude of waves in the circular basin, with generators (black) in the sector $(y<0)$ and absorbers (red) in $(y>0)$. The waves propagate in the $+y$-direction $\left(\beta=90^{\circ}\right)$. The upper left plot (a) shows the standing-wave system without absorption. The absorbers are (b) kinematic, (c) dynamic with 2D added-mass and damping forces, and (d) dynamic with oblique added-mass and damping forces. 

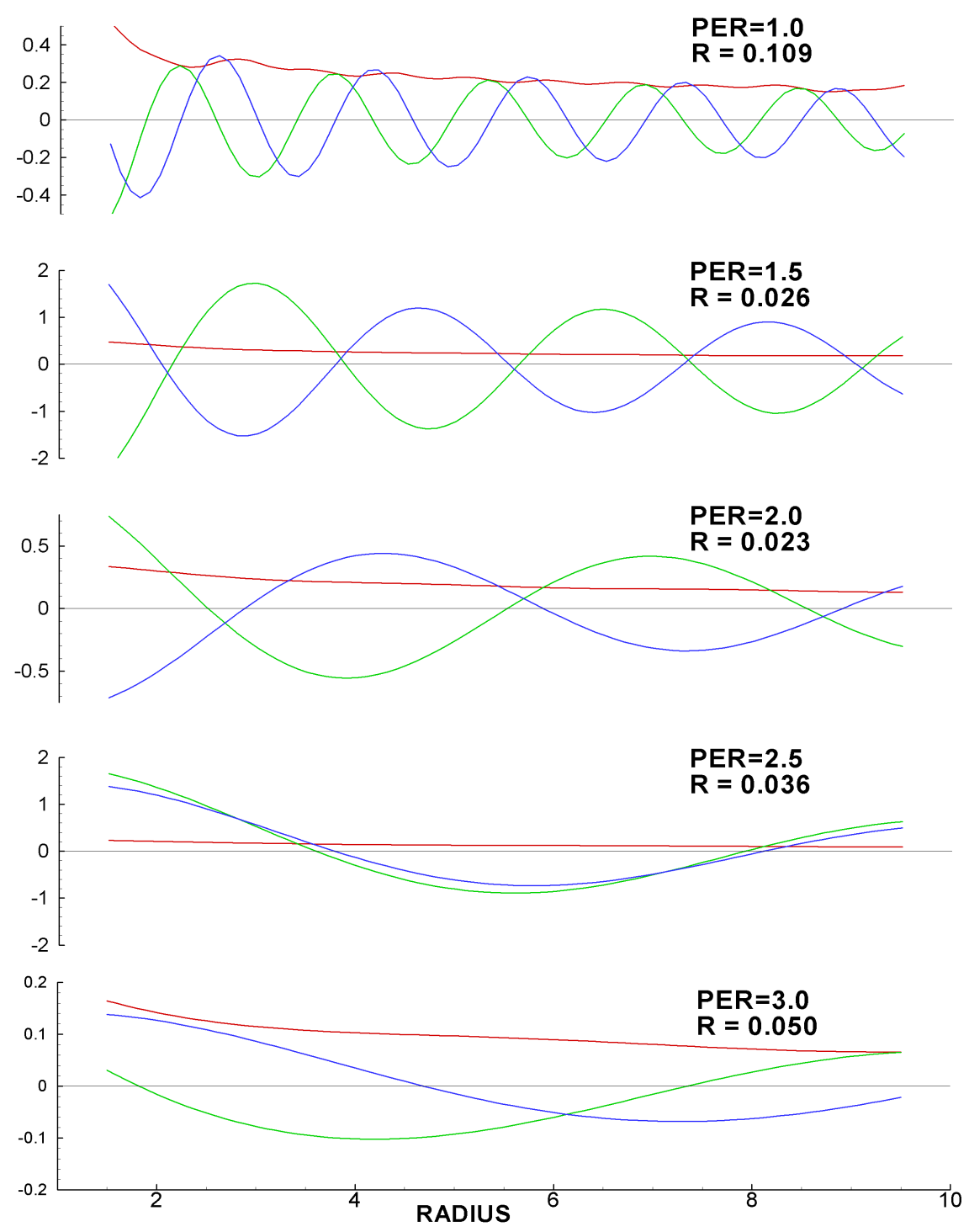

Figure 5: Plots showing the amplitudes of the standing waves in the circular basin, generated by heaving motion of the hemisphere (green) and axisymmetric motion of the wavemaker (blue) for different periods in seconds (PER). The amplitude of the wavemaker motion is reduced by a factor of $(1 / 10)$ for convenience in plotting. The red lines represent the amplitude of the radiating wave from the hemisphere with optimum absorption by the wavemaker. The abscissa is the radial distance $r$ from the center of the basin. The reflection coefficient $R$ is defined by the relation $R=\left(\eta_{\max }-\eta_{\min }\right) /\left(\eta_{\max }+\eta_{\min }\right)$ where $\left(\eta_{\max }, \eta_{\min }\right)$ are the maximum and minimum values of the radiated wave amplitude, multiplied by $\sqrt{\mathrm{kr}}$, in the range between $1.5 \mathrm{~m}$ and $9.5 \mathrm{~m}$ radius from the center. The hemisphere radius is $1 \mathrm{~m}$ and the basin radius is $10 \mathrm{~m}$. 


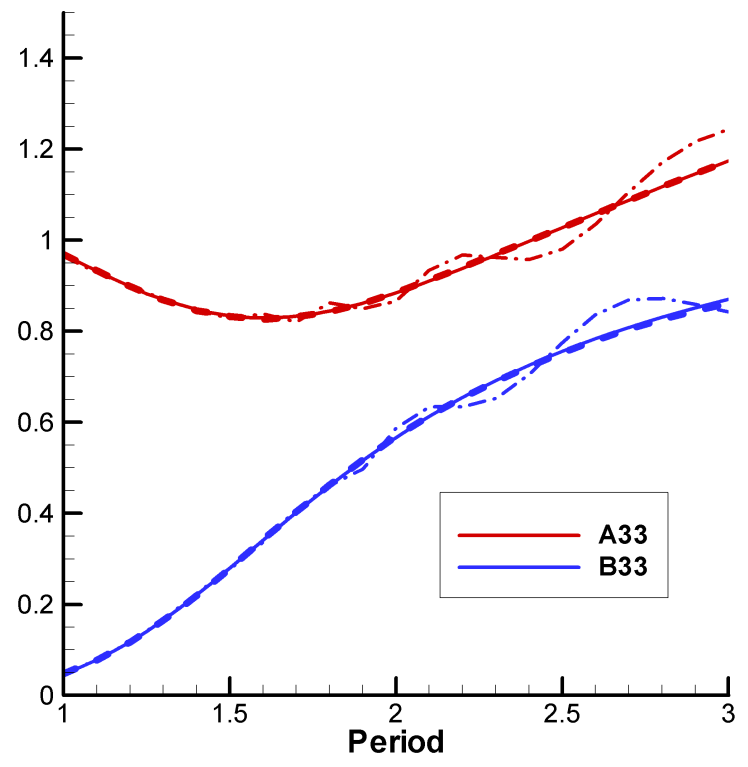

Figure 6: Added mass (red upper lines) and damping (blue lower lines) for the heaving hemisphere. The dashed lines are based on the optimum absorber amplitude derived from the free-surface elevations, using (35). The dot-dash lines are from computations with dynamic control of the wave absorber. The solid lines are from computations in open water. The added mass is normalized by $\rho$ and the damping by $\rho \omega$. The abscissa is the wave period in seconds.

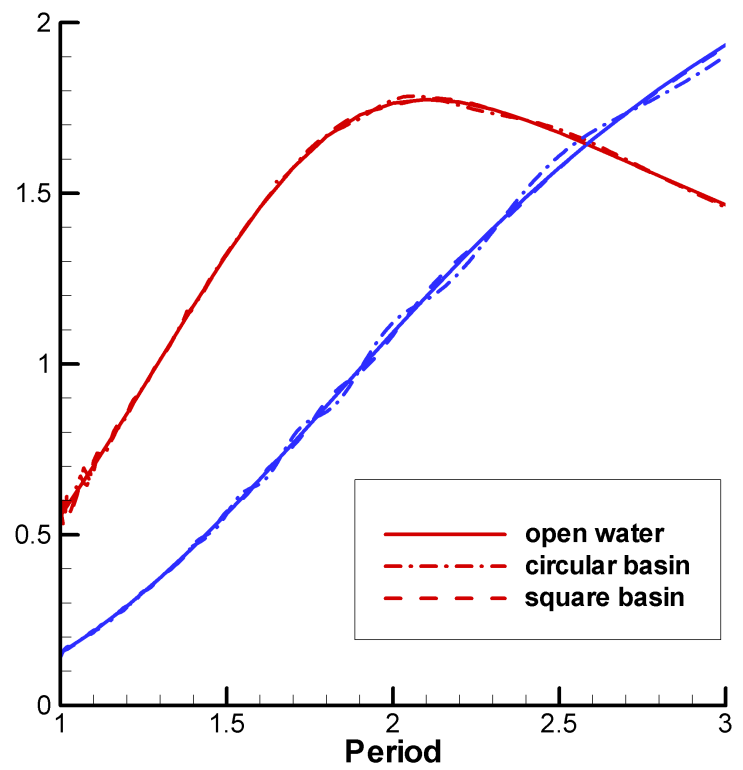

Figure 7: Exciting forces for the hemisphere in surge (red upper left lines) and heave (blue lower left lines). The dashed lines (square basin) and dot-dashed lines (circular basin) are based on computations using kinematic control to absorb the incident waves and dynamic control to absorb the scattered waves. The solid lines are from computations in open water. The exciting forces are normalized by $\rho g A$, where the wave amplitude $A$ is evaluated at the center of the basin without the hemisphere. The abscissa is the wave period in seconds. 


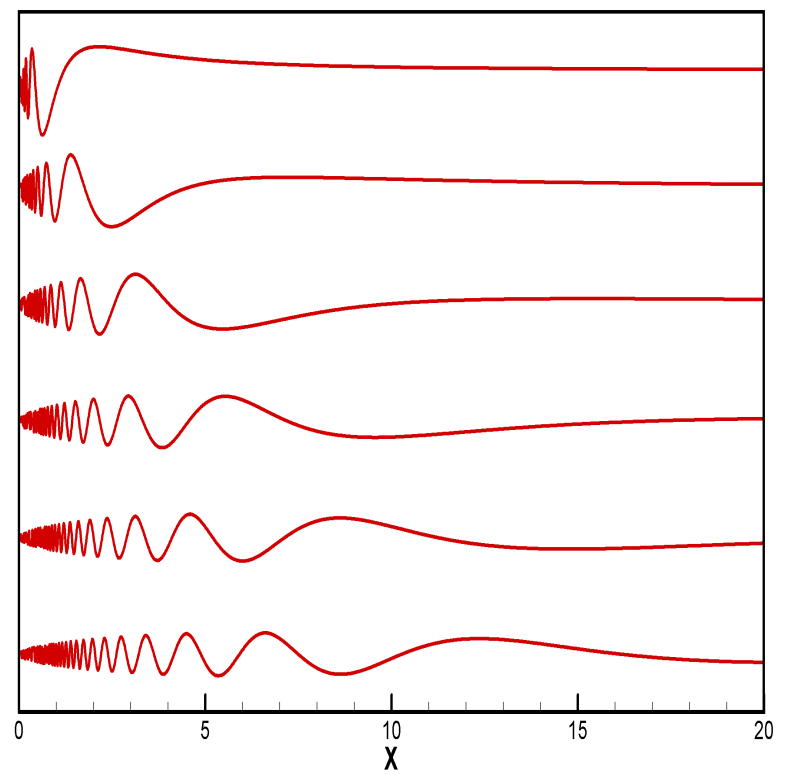

Figure 8: Plots of the influence function (40) at a sequence of time steps $t$ between 1 (top) and 6 (bottom) seconds. The distance $x$ is in meters.

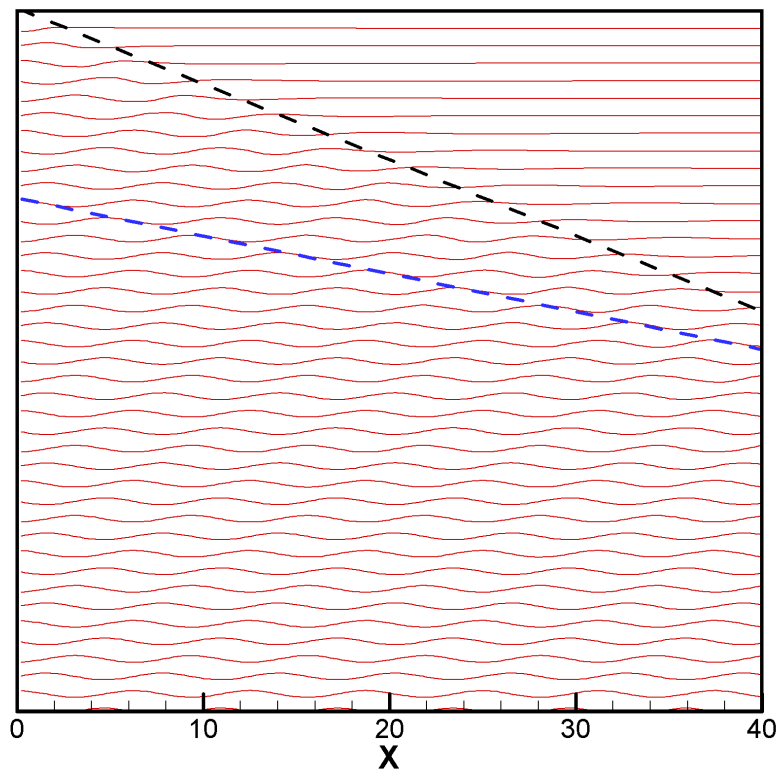

Figure 9: Waves generated by the wavemaker at $x=0$, at a sequence of time steps $t$ between 1.5 (top) and 60 (bottom) in steps of 1.5 seconds. The wave period is 2 seconds. The slopes of the upper and lower dashed lines are equal to the group velocity and phase velocity, respectively. 


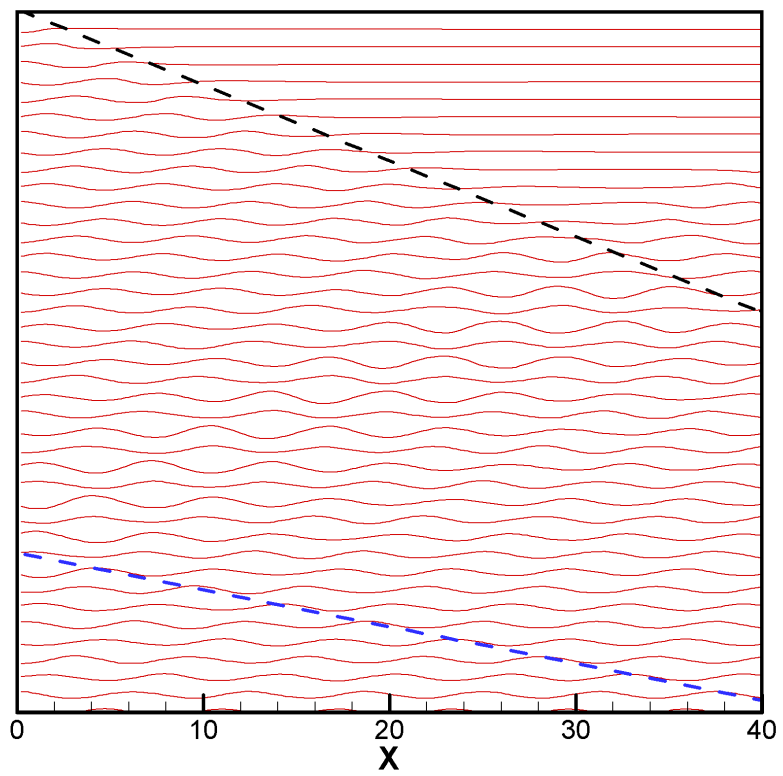

Figure 10: Waves generated by the wavemaker at $x=0$, with an image wavemaker at $x=80 \mathrm{~m}$ and an absorber at $x=40 \mathrm{~m}$, to simulate absorption in a basin of length $L=40 \mathrm{~m}$. The slopes of the upper and lower dashed lines are equal to the group velocity and phase velocity, respectively.

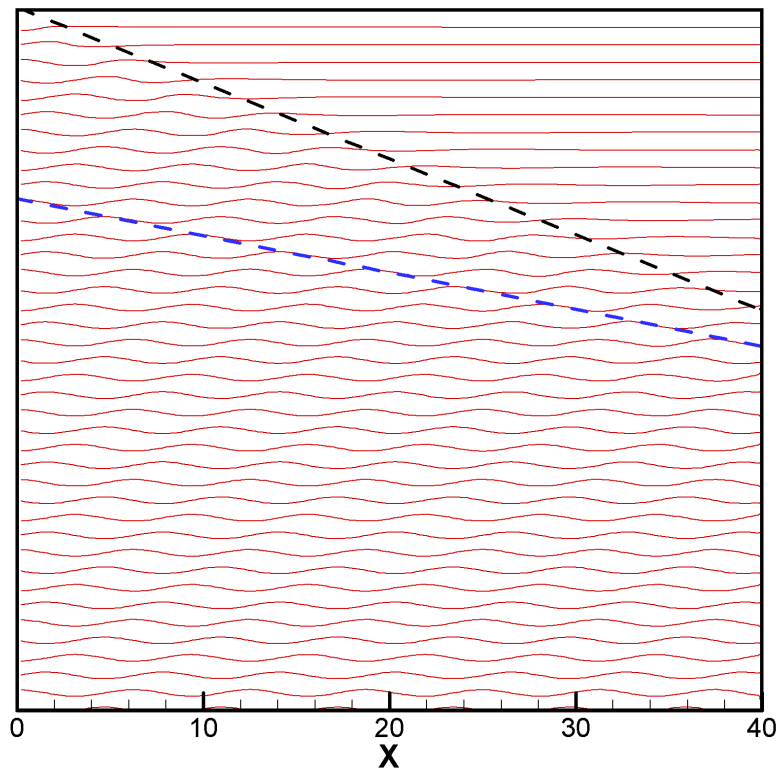

Figure 11: Waves generated by the wavemaker at $x=0$, with an absorber at $x=40 \mathrm{~m}$. The velocity of the absorber is proportional to the elevation of the incident wave at the absorber, and the resulting waves are defined by (41). The slopes of the upper and lower dashed lines are equal to the group velocity and phase velocity, respectively. 